\title{
CARACTERIZAÇÃO DOS DISTÚRBIOS COGNITIVOS NA DOENÇA DE PARKINSON
}

\section{Characterizing cognitive disorders in Parkinson's disease}

\author{
Mônica Maria de Azevedo Mello Carvalho Galhardo (1), Ana Karênina de Freitas Jordão do Amaral (2), \\ Ana Cláudia de Carvalho Vieira ${ }^{(3)}$
}

\begin{abstract}
RESUMO
Tema: comprometimentos cognitivos na doença de Parkinson. Objetivo: ressaltar a existência do déficit cognitivo na Doença de Parkinson, descrevendo as características dos comprometimentos cognitivos. Conclusões: as alterações cognitivas na Doença de Parkinson (referentes a memória, linguagem, capacidade visuo-espacial e funções executivas) são constatadas na literatura, no entanto, há uma carência de informações quanto à reabilitação fonoaudiológica de pacientes nessa área específica da linguagem.
\end{abstract}

DESCRITORES: Doença de Parkinson; Memória; Linguagem; Cognição

\section{INTRODUÇÃO}

A Doença de Parkinson (DP) foi descrita pela primeira vez em $1817^{1-4}$ por James Parkinson. Ao descrever a DP inicialmente a denominou "paralisia agitante", sendo esta enfermidade caracterizada pela presença de movimentos tremulantes involuntários, diminuição da força muscular, tendência à inclinação do corpo para frente e alteração da marcha (festinação). Os sentidos e o intelecto estavam preservados ${ }^{2,4}$, no entanto, na descrição dos casos relatados por James Parkinson haviam elementos que, se reconsiderados, correspondiam a indubitável comprometimento das funções cognitivas ${ }^{4}$.

(1) Fonoaudióloga do Centro de Reabilitação Francisco Loureiro, Jaboatão dos Guararapes, PE e do Centro de Atenção Psicossocial Ciranda de Vida do Cabo de Santo Agostinho, PE; Especialização em Linguagem: enfoque em Neurociências pela Faculdade Integrada do Recife; Especialista em Motricidade Orofacial.

(2) Fonoaudióloga do Centro de Reabilitação Francisco Loureiro, Jaboatão dos Guararapes, PE; Especialista em Motricidade Oral; Especialista em Gerontologia pela Universidade Federal da Paraíba; Mestre em Patologia/Morfologia Aplicada pela Universidade Federal de Pernambuco.

(3) Fonoaudióloga do Hospital da Restauração, Recife, PE; Mestre em Neuropsiquiatria e Ciências do Comportamento pela Universidade Federal de Pernambuco; Doutoranda em Neuropsiquiatria e Ciências do Comportamento pela Universidade Federal de Pernambuco.

Conflito de interesse: inexistente
Do ponto de vista patológico, a doença se caracteriza pela degeneração da substância negra, com perda progressiva de neurônios dopaminérgicos pigmentados e pela presença de neurônios contendo os corpos de Lewy ${ }^{5,6}$.

Charcot, neurologista francês, considerou como característica da DP o tremor na fase inicial da doença, ressaltando sua presença no repouso, de forma unilateral, afetando o membro superior. Fato importante é que seus estudos destacavam a presença de demência em alguns casos além de outros comprometimentos relatados ${ }^{2}$.

Dessa forma o objetivo deste estudo foi ressaltar a existência do déficit cognitivo na Doença de Parkinson, descrevendo as características dos comprometimentos cognitivos, destacando as alterações da memória, linguagem, capacidade visuoespacial e funções executivas, visto que esta relação DP e funções cognitivas é um assunto pouco abordado na literatura.

\section{MÉTODOS}

Foi realizado um levantamento bibliográfico dirigido ao tema Doença de Parkinson e Funções cognitivas, em periódicos não indexados e indexados na base de dados do Lilacs e Medline e por meio de folhetos informativos da Associação Brasil Parkinson, enfatizando as citações referidas desde 1976 até estudos mais recentes (2006). 
As palavras e descritores pesquisados foram: Doença Parkinson, memória, linguagem e distúrbio cognitivo.

Foi realizada uma análise do assunto com a finalidade de discutir criticamente as características clínicas da DP, enfatizando as alterações de linguagem, memória, capacidade visuo-espacial e funções executivas.

\section{REVISÃO DA LITERATURA}

\section{Características da DP}

A DP constitui-se numa desordem neurodegenerativa e progressiva ${ }^{1,3,7,8}$ de etiopatogenia desconhecida ${ }^{1,7}$, que acomete os neurônios dopaminérgicos da substância negra (pars compacta), resultando em um déficit de dopamina no corpo estriado ${ }^{1,8,9}$, substância negra e regiões profundas do cérebro ${ }^{6,8}$.

Caracteriza-se pela presença de disfunção monoaminérgica múltipla, incluindo o déficit de sistemas dopaminérgicos, colinérgicos, serotoninérgicos e noradrenérgicos ${ }^{7}$. O déficit dessas substâncias (neurotransmissores) afeta a capacidade do organismo de controlar os movimentos normais ${ }^{6,9}$.

Embora a DP habitualmente acometa indivíduos a partir dos 60 anos, têm sido descritos casos de início precoce ${ }^{10}$. Atinge $0,1 \%$ da população geral e $1 \%$ da população acima de 65 anos; sua incidência cresce com o envelhecimento. A sobrevida após o surgimento da doença é de 9 a 12 anos e trata-se de uma das principais causas de incapacidade da atividade motora em idosos ${ }^{9}$.

Por não ter uma etiologia definida, a DP é tida como idiopática (DPI) ${ }^{7}$, pois a razão pela qual um indivíduo desenvolve a DP permanece obscura, o fator desencadeador primário da morte celular nigral na DP permanece desconhecido. Apesar do enorme conhecimento da neuroquímica, dos mecanismos fisiopatológicos e da etiopatogenia da DP, ainda não se descobriu um marcador biológico que pudesse ser utilizado em seu diagnóstico. Dessa maneira, o estudo clínico dos pacientes ainda é o fator essencial para o correto diagnóstico ${ }^{11}$.

Nos estágios iniciais da DP, o processo neurodegenerativo é quase totalmente localizado nas fibras dopaminérgicas que inervam o putâmen dorso-lateral, e as manifestações clínicas são principalmente motoras ${ }^{1}$.

No estágio inicial da DP (estágio 1) ocorre o acometimento do núcleo motor dorsal dos nervos glossofaríngeo e vago, além da zona reticular intermediária e do núcleo olfatório anterior. No estágio 2, existe o comprometimento adicional dos núcleos da rafe, núcleo reticular gigantocelular e do complexo do locus ceruleus. No estágio 3, observa-se o comprometimento da parte compacta da substância negra do mesencéfalo. Já nos estágios 4 e 5 há comprometimentos das regiões prosencefálicas, do mesocórtex temporal e de áreas de associação do neocórtex e neocórtex pré-frontal, respectivamente. No estágio 6, ocorre o comprometimento de áreas de associação do neocórtex, áreas pré-motoras e área motora primária ${ }^{7}$.

Com a progressão da doença, a perda de dopamina (DA) envolve todo o striatum e outras regiões do córtex cerebral, do tronco e da medula espinal ${ }^{1}$.

Dessa forma, devido o acometimento de áreas diversas do encéfalo e tronco, é possível concluir que vários sinais da DP podem estar relacionados. Estes sinais são chamados não-motores (distúrbio do sono, a disfunção cognitiva, a depressão) ${ }^{7}$.

Nem todos os pacientes com DP apresentam todos os sintomas ao mesmo tempo. A doença pode afetar cada um de maneira diferente, e, em alguns casos, podem passar-se muitos anos antes de uma incapacitação ou limitação significativa das atividades cotidianas ${ }^{3,6}$.

Clinicamente, a DP apresenta-se como tremor, rigidez, bradicinesia e instabilidade postural que pioram com a progressão da doença. O tremor é de repouso, com predomínio distal e usualmente assimétrico ${ }^{1,5,6,7,11}$, sendo que o mais característico é um movimento chamado de "contar moedas" ou "enrolar pílulas" dos dedos ou movimento de flexão/ extensão dos dedos e pulso. Apresenta piora substancial quando o paciente é submetido a estresse ou durante alguma tarefa cognitiva. Costuma melhorar durante o sono e tem resposta variável ao tratamento medicamentoso ${ }^{5,9}$.

A rigidez (resistência aos movimentos articulares passivos) encontra-se presente de forma assimétrica no início da doença, sendo mais acentuada no hemicorpo onde predomina o tremor. A bradicinesia representa a lentidão de movimentos, é o sintoma mais incapacitante da doença e está presente desde o início da DP, levando o paciente a dificuldades nas atividades de vida diária. A lentidão pode ocorrer no momento de iniciar um movimento (acinesia) ou durante todo o movimento (bradicinesia). A instabilidade postural costuma ocorrer em uma fase mais avançada da DP, em que se observa uma postura encurvada e cada vez mais flexionada para frente ${ }^{5,9}$.

Ocorrem também como manifestações precoces, diminuição da expressão facial (hipomimia), diminuição do piscamento ocular e hipofonia ${ }^{1,5,7}$. Observa-se diminuição do balançar de braços e o pé do lado mais comprometido tende a arrastar; a escrita torna-se cada vez menor (micrografia), a deglutição de saliva torna-se dificultosa e há presença de sialorreia ${ }^{5}$. 
O comprometimento motor se torna bilateral após três a cinco anos do diagnóstico inicial. As alterações posturais e de marcha costumam aparecer em fases tardias e constitui a maior causa de limitação, perda de equilíbrio e quedas ${ }^{1}$.

Os pacientes parkinsonianos, em uma fase mais avançada, evoluem com dificuldade progressiva para realizar funções simples relacionadas às atividades da vida diária, tornando-se cada vez mais dependentes ${ }^{1}$.

Associadas às complicações motoras (flutuações e discinesias) existem complicações não motoras, representadas pelas disautonomias (hipotensão ortostática, distúrbios gastrointestinais, distúrbios urinários, distúrbios sexuais, distúrbios da termoregulação e da sudorese, distúrbios sensitivos e da dor, dermatite seborréica, distúrbios do sono e distúrbios respiratórios) e por alterações neuropsiquiátricas (psicose induzida por drogas e distúrbios cognitivos) ${ }^{5,12}$.

Ainda é incerto o efeito que o tempo de duração dos sintomas da DP exerce sobre as funções neurocognitivas. No entanto, é possível observar a relação entre o tempo de evolução da doença e o desempenho dos pacientes nos testes de memória, percepção e solução de problemas. Uma vez que sendo a DP uma patologia progressiva e crônica é de se esperar que a piora progressiva das habilidades cognitivas ocorra com a evolução da doença ${ }^{5}$.

As alterações neuropsiquiátricas encontradas na DP constam de: alterações cognitivas/demência; depressão; alucinações/delírio/delirium; mania/ hipomania; hipersexualidade; ansiedade/crises de pânico; transtorno obsessivo-compulsivo ${ }^{4,13}$.

Após oito anos de seguimento da doença cerca de três quartos dos pacientes já apresentavam os sinais da demência ${ }^{14,15}$. O risco de desenvolver demência é duas vezes maior em indivíduos com DP do que em controles com mesma idade ${ }^{16}$. A incidência de demência na DP é cumulativa com o avançar da idade e, a este respeito, aos 85 anos o paciente portador de DP apresentará demência em $65 \%$ dos casos (esta chance parece ser independente do aumento da possibilidade de desenvolver demência com o aumento da idade observado na população geral) ${ }^{9}$.

Dados relevantes sobre o estudo da DP e demência detectaram que:

- Parkinsonianos com a doença instalada mais tardiamente tem risco maior que os mais jovens de desenvolver a demência ${ }^{16}$;

- Baixo nível educacional correlaciona-se positivamente com desenvolvimento de demência na DP, da mesma forma que o observado na doença de Alzheimer (DA) ${ }^{16}$;
- Em estudo longitudinal de longo prazo, baixo desempenho em testes verbais era fator preditivo para demência ${ }^{17}$;

- Duração da doença, tratamento com levodopa, apolopoproteína E4, sugerindo bases biológicas diferentes da DA, não são fatores de risco para demência em DP ${ }^{16}$.

Observa-se que os pacientes com início precoce da doença (< 40 anos) apresentam gravidade maior dos sinais e sintomas da demência do que os pacientes acima dos 50 anos. Vários estudos demonstram que os pacientes parkinsonianos que exibem distúrbios cognitivos são habitualmente mais idosos no início da doença e estão mais gravemente acometidos. Os efeitos que a idade do paciente e o tempo de início da doença exercem sobre a cognição não podem ser considerados como independentes, pois pode haver uma associação entre o declínio cognitivo e a idade. Apesar disso, vários estudos demonstram que o início tardio da doença pode representar fator preditivo para o declínio cognitivo que se segue ${ }^{5}$.

Distúrbios visuo-espaciais e lentificação de processos decisórios são alterações cognitivas isoladas que podem surgir precocemente na evolução da DP sem que representem à instalação de um quadro demencial. Diferentemente, o quadro demencial na DP instala-se em fases mais adiantadas na evolução da doença e tem como principais características a lentificação do processo cognitivo (bradifrenia), a apatia, o comprometimento da memória e das funções executivas frontais ${ }^{18}$.

As alterações cognitivas na DP são explicadas pelo fato de que além de projeções do córtex motor, o estriado recebe projeções de áreas corticais de associação, do córtex sensitivo. Atualmente cinco circuitos são bem definidos: o circuito motor, que se origina na área suplementar motora; o circuito oculomotor, com origem no córtex frontal na área 8 de Broadmann; e os circuitos dorsolateral, órbito-frontal lateral e do cíngulo anterior, todos originados no córtex pré-frontal. Todos os circuitos compartilham as mesmas estruturas: lobo frontal, estriado, globo pálido, substância negra e tálamo. São circuitos contíguos, mas que permanecem anatomicamente segregados ao longo da alça neuronal de cada circuito. Dos circuitos acima, os três originados no córtex pré-frontal não têm, aparentemente, função motora. Portanto, não é surpresa que muitos achados clínicos revelem o envolvimento dos núcleos da base em uma grande variedade de funções não motoras ${ }^{5}$.

Os circuitos fronto-corticais estão relacionados com vários distúrbios neuropsiquiátricos, como a síndrome da demência subcortical e com diversos distúrbios do comportamento. A disfunção desses 
circuitos justifica os mais diversos déficits cognitivos observados nos pacientes com distúrbios do movimento ${ }^{5}$.

As disfunções associadas aos núcleos da base sempre estiveram relacionadas essencialmente aos distúrbios do movimento. Além das alterações motoras, os pacientes com doenças extrapiramidais, como a DP, apresentam alterações da função intelectual, do humor e da personalidade. A natureza e a gravidade das alterações neuropsiquiátricas observadas nestes pacientes refletem a extensão do envolvimento de estruturas relevantes dos circuitos fronto-subcorticais ${ }^{5,6}$.

Os critérios da DSM - IV (Manual de Diagnóstico e Estatística de Doenças Mentais), normalmente empregados para caracterizar quadros demenciais, estão mais voltados para o diagnóstico de doença de Alzheimer desconsideram que uma grave dificuldade motora compromete a autonomia do paciente. De outro lado, a depressão, um dos critérios para exclusão de demência segundo o DSM - IV está frequentemente presente na DP. As dificuldades motoras nos parkinsonianos podem levar à superestimação do seu comprometimento cognitivo ${ }^{18}$.

A diferenciação entre demência e DP deve ser feita em relação as seguintes condições: depressão, confusão mental, demência dos corpos de Lewy, hidrocefalia de pressão intermitente e doença de Alzheimer. Os mecanismos neurobiológicos implicados na demência da DP ainda não estão completamente esclarecidos, mas há dados sugestivos de que devem participar desse processo o comprometimento das alças dopaminérgicas que partem do mesencéfalo (circuito fronto-estriatal não-motor); alteração das projeções colinérgicas para o córtex cerebral, elementos neuropatológicos do tipo Alzheimer e corpos de Lewy ${ }^{18}$.

A principal consequência da demência na DP é a restrição quanto ao uso de medicação antiparkinsoniana, pois as mesmas podem provocar efeitos colaterais neuropsiquiátricos. Dessa forma, o manejo do quadro demencial na DP envolve rigorosa seleção de antiparkinsonianos a serem empregados, evitando-se o uso de medicação anticolinérgica ${ }^{18}$.

O processo de memória envolve várias etapas: (1) codificação (recebimento da nova informação); (2) armazenamento de curta duração; (3) consolidação, (armazenamento do material codificado de forma permanente) e; (4) recuperação da informação estocada ${ }^{5}$.

O distúrbio de memória está entre as alterações mais frequentes observadas na DP, caracterizado pela dificuldade em recordar informações verbais recentemente aprendidas, devido a déficit na codificação de novas informações ou a uma dificuldade na capacidade de utilizar eficientemente a codificação semântica devido a problemas no processamento da informação. Outro aspecto também prejudicado nesta função é o comprometimento da memória para conteúdo não-verbal, que costuma ser maior do que para o conteúdo verbal. Neste aspecto, as dificuldades no desempenho de tarefas visuoespaciais e lentificação do processamento mental poderiam corroborar a dificuldade apresentada pelo paciente na memória não-verbal, principalmente no que concerne a reprodução de desenhos complexos e localização de lugares em mapa. Os pacientes com DP apresentam performance normal em várias medidas de memória explícita e de reconhecimento, mas apresentam comprometimento no teste cognitivo Torre de Toronto ${ }^{5}$.

Os distúrbios de linguagem e compreensão são de ocorrência rara nos pacientes parkinsonianos. $\mathrm{O}$ comprometimento mais observado envolve a capacidade de nomear e a fluência verbal. Quanto a fluência, é possível que ela esteja alterada devido a fatores relacionados à disartria, bradicinesia ou ao próprio comprometimento das funções executivas observados nos pacientes com DP ${ }^{5}$.

Os estudos da fluência verbal em pacientes parkinsonianos permanecem conflitantes, apesar de os achados estarem caracterizados como fatores de risco para o desenvolvimento da demência na DP 19 .

As habilidades visuo-espaciais são complexas e exigem a integração das funções corticais dos lobos occipitais, parietais e frontais, assim como a participação de estrutura subcorticais. A função visuo-espacial se divide em capacidade visual sensitiva, habilidade visual perceptiva (discriminação e reconhecimento), habilidades visuo-motoras, atenção visuo-espacial, cognição visuo-espacial e orientação espacial corporal. Apesar de ser uma divisão didática, é conveniente em termos de processos neuropsicológicos, considerando que cada uma destas atividades pode ser testada através de instrumentos diferentes. Na DP podem-se encontrar algumas destas atividades comprometidas, enquanto outras permanecem intactas ${ }^{5}$.

A percepção visual está dividida em capacidade discriminativa (capacidade de analisar um estímulo novo) e capacidade de reconhecer (capacidade de identificar um estímulo visual familiar). A tarefa de reconhecimento visual exige memória preservada para informação visual previamente aprendida e percepção visual acurada para interpretação. O reconhecimento visual encontra-se preservado nos pacientes com DP, e estes não costumam apresentar déficits de reconhecimento como agnosias, prosopagnosia ou agnosias do ambiente. No entanto, estão comprometidas as respostas que exigem habilidade visual discriminatória, como a orientação 
linear, desenhos complexos, percepção de posição espacial, percepção de constância de formas e tamanhos e relacionamento espacial. Os pacientes parkinsonianos também apresentam dificuldade para identificar figuras específicas envolvidas em padrões mais complexos ${ }^{20}$.

As funções executivas referem-se à habilidade cognitiva de organizar um comportamento como solução para um problema complexo, como aprender e organizar novas informações, formar conceitos, copiar figuras e buscar sistematicamente fatos memorizados. Está profundamente envolvida na ativação de memórias remotas, na independência das contingências ambientais, na mudança e manutenção apropriada de estratégias, geração e utilização adequada de programas motores e capacidade de utilizar a habilidade verbal para guiar o comportamento ${ }^{21}$.

Autores relatam a ocorrência de demência em cerca de $10 \%$ a $50 \%$ dos pacientes parkinsonianos, geralmente com comprometimento das funções visuo-espaciais e frequentemente acompanhado de alucinações ${ }^{1,9}$.

Considerando a íntima relação entre o núcleo da base e o córtex frontal, os pacientes com DP podem apresentar falhas em executar tarefas com alta demanda das funções executivas, principalmente quando a solução de problemas requer que o indivíduo planeje e execute uma estratégia usando apenas a fonte de dicas internas para guiar o comportamento. Estas alterações podem ser observadas desde o início da doença ou mesmo em pacientes ainda não tratados ${ }^{5}$.

A identificação de alterações cognitivas na DP oferece algumas dificuldades. As funções executivas, que representam os domínios cognitivos, geralmente afetados na DP, habitualmente não são avaliadas. São, portanto, necessários testes específicos para avaliar essas funções que compreendem: formação de conceitos, solução de problemas, capacidade (aptidão) para mudanças de padrões e elaboração de estratégias ${ }^{18}$.

De uma forma geral a demência associada à DP é caracterizada por: redução ou falta de iniciativa para atividades espontâneas; incapacidade de desenvolver estratégias exitosas para resolução de problemas; lentificação de processos mnésticos; lentificação do processamento global da informação; prejuízo da percepção visuo-espacial; dificuldades de conceitualização; dificuldades na geração de listas de palavras ${ }^{9}$.

A demência associada à DP, na ocasião do diagnóstico, geralmente é de grau leve a moderado e na exploração clínica encontram-se mais salientes: a lentificação psicomotora e do processamento cognitivo global, disfunção executiva (prejuízo na abstração, na formação de conceitos, na geração espontânea de palavras), as disfunções construtivas e um baixo rendimento em algumas tarefas matemáticas ${ }^{9}$.

A prevalência da demência na $D P$, segundo dados da literatura, oscila em torno de $20 \%$ a $40 \%$, variando de acordo com a metodologia empregada e as características da população ${ }^{18}$.

O levantamento bibliográfico realizado revelou que a DP apresenta uma gama variada de sintomas, tanto motores quanto cognitivos. No entanto, as alterações de ordem motora ganham um amplo destaque no meio científico em detrimento das alterações de ordem cognitiva.

A literatura fonoaudiológica aborda o tema essencialmente em seus aspectos motores, enfatizando em diversos estudos os aspectos relacionados à motricidade e à voz em detrimento da linguagem.

Apesar de poucos estudos relacionando a sintomatologia motora com a alteração das funções cognitivas na DP, os autores pesquisados referem que o paciente que demonstra melhor preservação cognitiva tem predomínio dos tremores. Diferentemente, aqueles nos quais predominam a bradicinesia e a rigidez, os sintomas são relacionados com maior comprometimento intelectual, principalmente nos testes de fluência verbal, memória visual, velocidade de processamento da informação e habilidade construcional.

Apesar da consistência dos achados de alterações cognitivas que afetam a linguagem do paciente, não foram encontrados muitos periódicos que abordassem a respeito do tratamento fonoaudiológico na DP na área da linguagem.

\section{CONCLUSÃO}

Diante da revisão realizada, foi detectada que as alterações das funções cognitivas são realidade na DP. Algumas alterações se refletem significativamente na linguagem. Apesar disso, há uma carência de literatura que aborde a intervenção fonoaudiológica.

Os estudos que relacionam as funções cognitivas e a DP demonstram alterações na memória, linguagem, capacidade visuo-espacial e funções executivas, e caracterizam a DP como uma demência, que muitas vezes irá manifestar os seus sintomas no decorrer de alguns anos após o paciente ser diagnosticado.

Apesar das alterações motoras se destacarem e serem apontadas como os sinais da DP, é importante saber que os efeitos das alterações cognitivas podem ser reabilitados e tratados de forma preventiva, por meio de uma terapia que estimule o uso dessas funções. 


\begin{abstract}
Background: cognitive commitments in Parkinson's disease. Purpose: to point out the existence of cognitive deficit in Parkinson's disease, describing its characteristics. Conclusions: cognitive alterations in Parkinson's disease (such as memory, language, visual-spatial capacity and execution functions) are found in the literature, however, there is a lack of information such as the speech therapist rehabilitation of patients in such -specific language-related area.
\end{abstract}

KEYWORDS: Parkinson Disease; Memory; Language; Cognition

\section{REFERÊNCIAS}

1. Scorza FA, Henriques LD, Albuquerque M. Doença de Parkinson: tratamento medicamentoso e seu impacto na reabilitação de seus portadores. Mundo Saúde. 2001; 25(4):365-70.

2. Barbosa ER, Teive HAG. Doença de Parkinson: aspectos históricos. In: Andrade LAF, Barbosa RE, Cardoso F, Teive HAG. Doença de Parkinson: estratégias atuais de tratamento. 2. ed. São Paulo: Segmento Farma; 2006. p. 11-6.

3. Aragão FA, Navarro FM. Influências do envelhecimento, do tempo de evolução da doença e do estado cognitivo sobre os episódios de quedas, em uma população parkinsoniana. Fisioterapia Brasil. 2006; 6(4):250-1.

4. Reich EG. Demencias subcorticales en enfermidades extrapiramidales. In: Mangone CA, Allegri RF, Arigaza RL, Ollari JA. Demência: enfoque multidisciplinario. Buenos Aires: Bayer; 1997. p. 157-82. 5. Rocha MSG. Doença de Parkinson: aspectos neurpsicológicos. In: Andrade VM, Santos FH, Bueno OFA, organizadores. Neuropsicologia hoje. São Paulo: Artes Médicas; 2004 p. 349-70.

6. Folhetos da Associação Brasil Parkinson. Quais são as minhas opções de tratamento: lidando com a doença de Parkinson. Folheto informativo.

7. Teive HA. Neuroproteção: fatos, mitos e quimeras. In: Andrade LAF, Barbosa RE, Cardoso F, Teive HAG. Doença de Parkinson: estratégias atuais de tratamento. 2. ed. São Paulo: Segmento Farma; 2006. p. 17-35.

8. Vitorino MR, Homem FCB. Doença de Parkinson: da fonação à articulação. Fono Atual. 2001; 4(17):35-9.

9. Caixeta L. Demências. São Paulo: Lemos Editorial; 2004.

10. Hoehn MM. Age distribution of patients with parkinsonism. J Am Geriatr Soc. 1976; 24(2):79-85.
11. Andrade LAF. Estratégia no tratamento do paciente com início precoce. In: Andrade LAF, Barbosa ER, Cardoso F, Teive HAG. Doença de Parkinson: estratégias atuais de tratamento. 2. ed. São Paulo: Segmento Farma; 2006. p. 65-97.

12. Teive HA. Manejo das complicações nãomotoras da doença de Parkinson: disautonomias. In: Andrade LAF, Barbosa ER, Cardoso F, Teive HAG. Doença de Parkinson: estratégias atuais de tratamento. 2. ed. São Paulo: Segmento Farma; 2006. p.131-41.

13. Nuti A, Ceravolo R, Piccinni A, Dell'Agnello G, Bellini G, Gambaccini G, et al. Psychiatric comorbidity in a population of Parkinson's disease patients. Eur J Neurol. 2004; 11(5):315-20.

14. Barbosa ER. Agonistas dopaminérgicos. In: Andrade LAF, Barbosa ER, Cardoso F, Teive HAG. Doença de Parkinson: estratégias atuais de tratamento. 2. ed. São Paulo: Segmento Farma; 2006. p. 37-49.

15. Aarsland D, Andersen K, Larsen JP, Lolk A, Kragh-Sorensen P. Prevalence and characteristics of dementia in Parkinson disease: an 8-year prospective study. Arch Neurol. 2003; 60(3):387-92. 16. Marder K, Cote L, Tang M. The risk and predictive factors associated with dementia in Parkinson's disease. In: Korczyn A. Demencia in Parkinson's disease. Bologna: Monduzzi; 1994. p. 51-4.

17. Jacobs DM, Marder K, Cote LJ, Sano M, Stern $Y$, Mayeux R. Neuropsychological characteristics of preclinical dementia in Parkinson's disease. Neurol. 1995; 45(9):1691-6.

18. Barbosa ER. Tratamento das complicações neuropsiquiátricas na doença de Parkinson. In: Andrade LAF, Barbosa RE, Cardoso F, Teive HAG. Doença de Parkinson: estratégias atuais de tratamento. 2. ed. São Paulo: Segmento Farma; 2006. p. 143-53. 
19. Mahieux F, Fenelon G, Flahault A, Manifacier M, Michelet D, Boller F. Neuropsychological prediction of dementia in Parkinson's disease. J Neurol Neurosurg Psychiatr. 1998; 64(2):178-83.

20. Levin BE. Spatial cognition in Parkinson disease. Alzheimer Dis Assoc Disord. 1990; 4(3):161-70.
21. Chow T, Cummings JL. Frontal subcortical circuits. The Human Frontal Lobes: Functions and disorders. New York: The Guilford Press; 1999. p. 3-26.

RECEBIDO EM: 02/07/2007

ACEITO EM: 07/11/2008

Endereço para correspondência:

Mônica Maria de Azevedo Mello Carvalho

Galhardo

Rua Manoel Graciliano de Souza, 862/102

Olinda - PE

CEP: $53140-160$

E-mail: monicamcarvalho@yahoo.com.br 